\title{
采用单节变形履带机构的新型轮椅机器人*
}

\author{
于苏洋 ${ }^{1,2}$ 王 $\quad$ 挺 $^{1}$ 王志东 ${ }^{1,3}$ 王越超 ${ }^{1}$ 姚 辰 $^{1} \quad$ 李小凡 $^{1}$ \\ (1. 中国科学院沈阳自动化研究所机器人学国家重点实验室 沈阳 110016;
}

2. 中国科学院大学 北京 100049 ;

3. 千叶工业大学先进机器人技术部 千叶 275-0016 日本)

\begin{abstract}
摘要: 提出一种采用单节变形履带机构的轮椅机器人。该机构能够通过两组摆臂的转动对履带形状与张紧力进行主动控制, 以使履带形状更好地与障碍物相适应, 从而提高传统轮椅的越障能力。在对比现存各种轮椅机器人越障机构特点的基础上, 介绍这种新型轮椅机器人的机构方案及对楼梯这种典型障碍物的爬越过程。针对爬越楼梯这一越障运动, 将轮椅机器人转换 成等效串联机器人, 建立用于描述机器人位置及履带形状的几何模型, 并给出基于拉格朗日动力学方程的机器人主动构件驱 动力矩的优化求解方法。通过仿真得到了轮椅机器人爬越楼梯过程中各主动构件驱动力矩的变化情况, 进而指导完成了机器 人驱动系统与机械结构设计并制作出原理样机。通过样机爬越楼梯试验验证了轮椅机器人的越障能力。
\end{abstract}

关键词: 轮椅机器人 单节变形履带机构 越障能力 驱动力矩

中图分类号: TP242

\section{Wheelchair Robot Equipped with Variable Geometry Single Tracked Mechanisms}

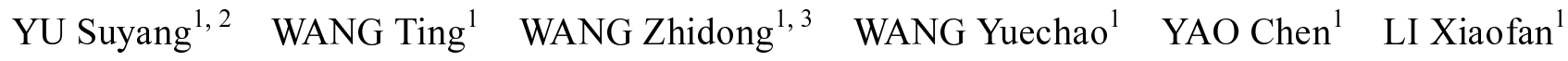 \\ (1. State Key Laboratory of Robotics, Shenyang Institute of Automation, \\ Chinese Academy of Sciences, Shenyang 110016; \\ 2. University of Chinese Academy of Sciences, Beijing 100049; \\ 3. Department of Advanced Robotics, Chiba Institute of Technology, Chiba 275-0016, Japan)
}

\begin{abstract}
A wheelchair robot equipped with variable geometry single tracked mechanisms(VGSTMs) is proposed. This mechanism can actively control the track shape and tension to adapt to the obstacles by rotating the two pairs of flippers, so it becomes possible to improve the obstacle clearing capability of the traditional wheelchair. Following a presentation of some typical mechanisms for obstacle clearing, the mechanism scheme and the stair-climbing process of the robot are introduced. With the aim of stair-climbing, the wheelchair robot is translated to a serial manipulator, base on which the geometric model that can describe the position and the track shape of the robot is established, and the optimal solution method of the driving component moment with Lagrange dynamic equations is proposed. In the simulation, the variations of the driving moment during stair-climbing are obtained, and then the design of the driving system and the mechanical structure of the robot are completed with the simulation results. The stair-climbing experiment is performed with the prototype to verify the obstacle clearing capability of the robot.
\end{abstract}

Key words: Wheelchair robot Variable geometry single tracked mechanism Obstacle clearing capability Driving moment

\section{0 前言}

轮椅是下肢行动不便者重要的代步工具。传统 轮椅在平地上运动比较灵活, 但当遇到障碍物时其 运动会受到很大的限制, 给使用者带来了极大的不

* 国家自然科学基金资助项目(60805048)。20121206 收到初稿, 20130506 收到修改稿
便。为了提高传统轮椅的越障能力, 尤其是对日常 建筑物中广泛存在的楼梯的爬越能力, 国内外很多 研究者尝试将移动机器人的越障机构与传统轮椅相 结合, 研制轮椅机器人。

在国内外现有移动机器人所采用的越障机构 中, 具有较强的越障能力尤其是能够实现爬越楼梯 这种越障运动的主要有星形轮式机构、腿式机构和 履带式机构三种。星形轮式轮椅机器人虽然能够完 
成爬越楼梯的运动, 但爬越过程中本体的波动会降 低乘坐的舒适度 ${ }^{[1-3]}$ 。同时, 如果机器人采用单组星 形轮式机构, 在无外力辅助时爬越的安全性较差 ${ }^{[1]}$; 而采用单组星形轮结合辅助机构或多组星形轮式机 构又会使机器人的体积过于庞大 ${ }^{[2-3]}$ 。腿式机构应用 在轮椅机器人上能为其带来较强的越障能力, 但同 时机器人的机构与控制也较为复杂 ${ }^{[4-5]}$ 。相比之下, 履带式机构因其接地面较大, 能使轮椅机器人爬越 楼梯过程具有较高的安全性, 同时机器人的机构也 比较简捷。但采用传统单节履带机构的轮椅机器人 对障碍物的适应能力非常有限, 运动在楼梯顶端时 不能在无外力辅助情况下实现本体姿态的平稳转 换 ${ }^{[6]}$ 。为了解决单节履带式轮椅机器人对楼梯顶端 适应性差的问题, 一些轮椅机器人采用了多节变形 履带或与其原理相似的单节履带结合辅助摆臂的机 构方案 ${ }^{[7-9]}$ 。这类机构能够使轮椅机器人避免在楼梯 顶端出现本体姿态突变的现象, 但姿态变化的平缓 性仍有待提高。

在此情况下, 在移动机器人领域有所应用的各 种单节变形履带机构, 尤其是能够同时对履带形状 与张紧力进行主动控制的单节变形履带机构因其具 备更强的将履带形状主动与障碍物相适应的能 力 $^{[10-13]}$, 为进一步提高履带式轮椅机器人的越障能 上力提供了一种新的选择。因此, 本文首先提出了 一种采用单节变形履带机构的轮椅机器人的机构方 案, 并介绍了机器人爬越楼梯的过程; 之后建立了 机器人爬越楼梯过程的几何模型与动力学模型, 提 出了机器人主动构件驱动力矩优化求解方法并通过 仿真得到了主动构件驱动力矩在机器人爬越楼梯过 程中的变化情况; 最后在仿真结果指导下完成了机 器人驱动系统与机械结构设计, 并制作出原理样机 进行了机器人爬越楼梯试验。

\section{1 机器人机构方案及爬越楼梯过程}

\section{1 机构方案}

这种新型轮椅机器人的机构方案如图 1 所示。 机器人机构由上方固定有座椅的车架和在车架两侧 对称布置的单节变形履带机构组成。在变形履带机 构中, 一组前摆臂和一组后摆臂分别可以同步驱动 用来同时控制机器人履带的形状和张紧力。两组摆 臂的末端分别安装有行星轮, 其中两个后摆臂行星 轮分别可以独立驱动用来控制机器人的行走与转 向。在机器人机构中还设置有一些诱导轮、压带轮 以及承重轮, 用来对履带进行支撑。

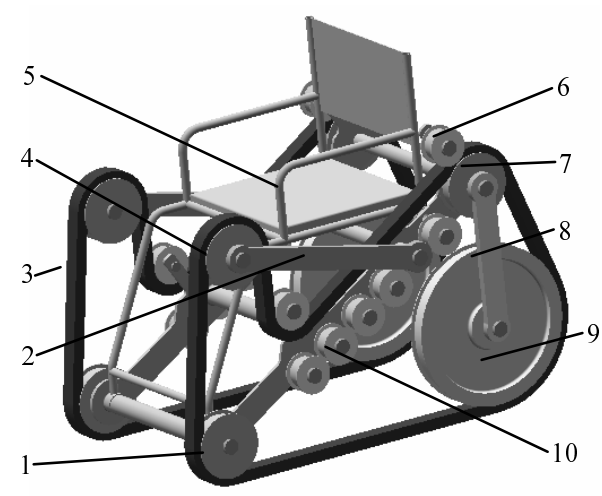

(a) 后摆臂转动到初始位置

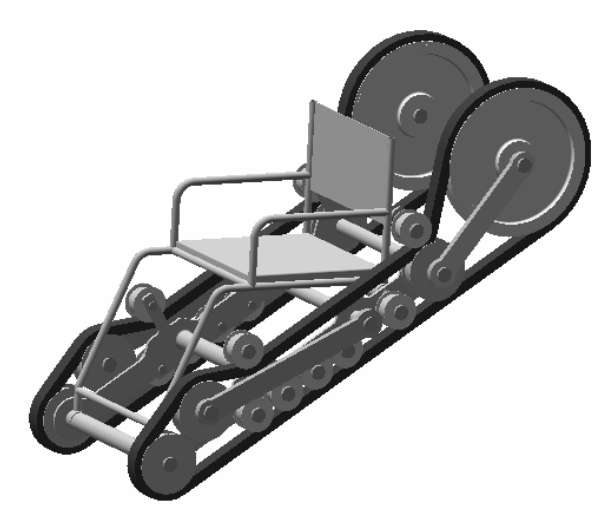

(b) 后摆臂转动到极限位置

图 1 轮椅机器人机构方案

1. 前承重轮 2. 前摆臂 3. 履带 4. 前摆臂行星轮 5. 车架及座椅 6. 压带轮 7. 诱导轮 8. 后摆臂 9. 后摆臂行星轮 10 . 极限位置承重轮

\section{2 爬越楼梯过程}

这种新型轮椅机器人爬越楼梯的过程可以划 分为四个阶段。第一阶段机器人背向楼梯, 前、后 摆臂逆时针转动使座椅有一定的后倾(图 2a)。第二 阶段机器人开始爬越前几级台阶。在机器人运动的 同时, 前、后摆臂根据需要继续逆时针转动以保持 合适的座椅倾角(图 2b、2c)。第三阶段机器人完全 进入楼梯, 并以固定的履带形状在楼梯上运动(图 $2 \mathrm{~d}$ )。当机器人后摆臂全部伸出楼梯时, 第四阶段开 始。此时机器人先停止向上运动, 后摆臂顺时针转 动直到后摆臂行星轮支撑到楼梯顶面, 使履带产生 适应楼梯顶端的变形(图 2e、2f)。之后机器人重新 开始向上运动, 同时前、后摆臂继续顺时针转动直 到机器人完全运动到楼梯顶面(图 $2 \mathrm{~g} 、 2 \mathrm{~h}$ )。

\section{2 几何模型}

\section{1 等效串联机器人}

在忽略履带质量, 保留作用在各履带轮两侧的 履带张紧力的情况下, 轮椅机器人可以转化为图 3 


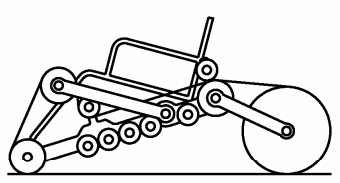

(a)

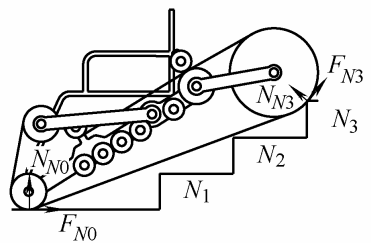

(c)

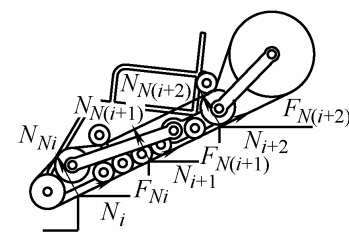

(e)

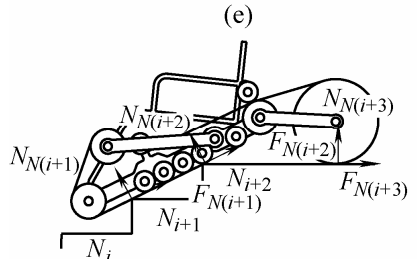

(g) (b)

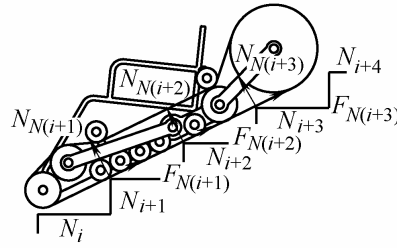

(d)

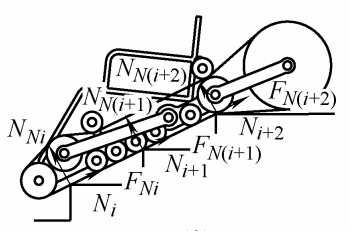

(f)

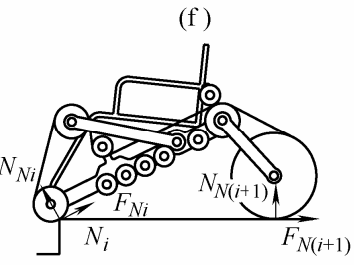

(h)

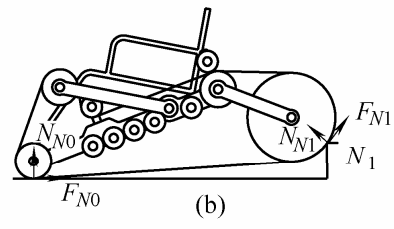

图 2 轮椅机器人爬越楼梯过程

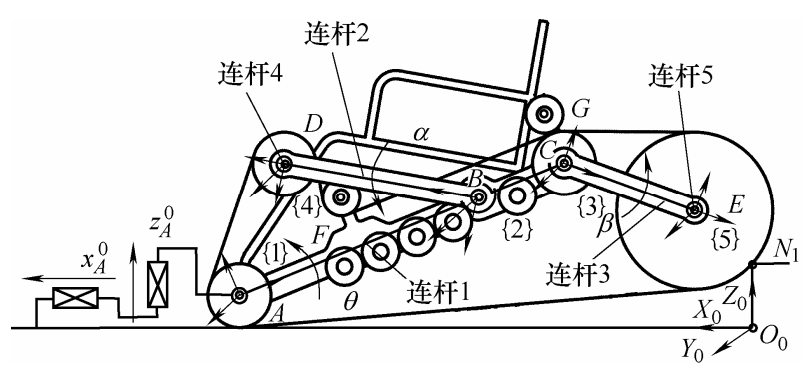

图 3 等效串连机器人

所示的等效串联机器人, 图 3 中 $F_{N I}$ 与 $N_{N I}$ 为履带与 楼梯间作用力, 后文中有详述。

图 3 中定义点 $A \sim G$ 及 $N_{i}$ 分别指代轮椅机器人 前承重轮、前后摆臂转动关节、前后摆臂行星轮、 压带轮、诱导轮等关键构件以及楼梯第 $i$ 级台阶支 点的位置; 串连机器人的各连杆分别与轮椅机器人 的车架(包括假设与其固定的乘坐者)、前、后摆臂 以及前、后摆臂行星轮等效; 串连机器人的各关节 分别描述轮椅机器人车架的平移与转动以及前、后 摆臂的转动; 坐标系 $\{k\}(k=0 \sim 5)$ 分别与楼梯底面以 及串联机器人的各连杆固连。进而在给定轮椅机器 人机构参数的基础上, 用来描述轮椅机器人的位置 及履带形状的关节变量可以表示为

$$
\boldsymbol{q}=\left(\begin{array}{lllll}
x_{A}^{0} & z_{A}^{0} & \theta & \alpha & \beta
\end{array}\right)^{\mathrm{T}}
$$

式中 $\left(x_{A}^{0}, z_{A}^{0}\right)$ 一一轮椅机器人前承重轮轮心在坐

\section{2 几何约束方程}

标面 $X_{0} O_{0} Z_{0}$ 内的坐标

$\theta$ 一一轮椅机器人座椅倾角

$\alpha, \beta$ 一一轮椅机器人前、后摆臂转角

轮椅机器人在爬越楼梯过程中, 前、后摆臂的 转动要受到楼梯位置和履带长度的约束。利用这两 个约束条件推导出的几何约束方程, 可以描述机器 爬越楼梯过程中的运动位置、座椅倾角以及前、后 摆臂转角之间的关系。

推导过程主要针对轮椅机器人与楼梯台阶接 触后的爬越过程第二、第三和第四阶段，且根据指 导机构设计这一任务需求作出如下简化: 第二阶段 针对机器人后摆臂行星轮支撑在楼梯台阶支点的情 况, 第三阶段针对机器人后摆臂转动到极限位置的 情况, 第四阶段针对机器人后摆臂行星轮支撑到楼 梯顶面之后的情况。

首先根据楼梯位置对轮椅机器人的约束情况, 推导出反映机器人所处位置、座椅倾角及后摆臂转 角之间关系的楼梯位置约束方程如下。

第二阶段(图 2b、2c)

$$
f_{S P 2}\left(x_{A}^{0}, z_{A}^{0}, \theta, \beta\right)=\left(x_{E}^{0}-x_{N i}^{0}\right)^{2}+\left(z_{E}^{0}-z_{N i}^{0}\right)^{2}-r_{3}^{2}=0
$$

第三阶段(图 2d)

$$
f_{S P 3}\left(x_{A}^{0}, z_{A}^{0}, \theta, \beta\right)=\frac{(\tan \varphi) x_{E}^{0}+z_{E}^{0}-h}{\sqrt{(\tan \varphi)^{2}+1}}-r_{3}=0
$$

第四阶段(图 $2 \mathrm{~g} 、 2 \mathrm{~h}$ )

$$
f_{S P 4}\left(x_{A}^{0}, z_{A}^{0}, \theta, \beta\right)=z_{E}^{0}-\left(z_{N i}^{0}+r_{3}\right)=0
$$

式中 $\left(x_{E}^{0}, z_{E}^{0}\right)$ 一一后摆臂行星轮轮心在坐标面 $X_{0}$ $O_{0} Z_{0}$ 内的坐标

$\left(x_{N i}^{W}, z_{N i}^{W}\right)$ 一楼梯台阶支点在坐标面 $X_{0} O_{0} Z_{0}$ 内的坐标

$r_{3}$ 一一后摆臂行星轮半径

$h$ 一一楼梯台阶高度

$\varphi$ 一一楼梯倾角

之后根据履带长度对轮椅机器人的约束情况, 推导出反映机器人所处位置、座椅倾角及前、后摆 臂转角间关系的履带形状约束方程如下。

机器人底面履带为直线段(图 $2 b 、 2 c 、 2 d)$

$$
\left\{\begin{array}{c}
f_{T S 11}=L_{T}-\angle A \times r_{2}-L_{A D}-\angle D \times r_{2}-L_{D F}-\angle F \times r_{1}- \\
\quad L_{C F}-\angle C \times r_{2}-L_{C E}-\angle E \times r_{3}-L_{A E}=0 \\
f_{T S 12}=L_{T}-\angle A \times r_{2}-L_{A D}-\angle D \times r_{2}-L_{D F}-\angle F \times r_{1}- \\
L_{F G}-\angle G \times r_{1}-L_{E G}-\angle E \times r_{3}-L_{A E}=0
\end{array}\right.
$$


机器人底面履带为折线段(图 $2 \mathrm{~g} 、 2 \mathrm{~h}$ )

$\left\{\begin{array}{c}f_{T S 21}=L_{T}-\angle A \times r_{2}-L_{A D}-\angle D \times r_{2}-L_{D F}-\angle F \times r_{1}- \\ L_{C F}-\angle C \times r_{2}-L_{C E}-\angle E \times r_{3}-L_{A N i}-L_{E N i}=0 \\ f_{T S 22}=L_{T}-\angle A \times r_{2}-L_{A D}-\angle D \times r_{2}-L_{D F}-\angle F \times r_{1}- \\ L_{F G}-\angle G \times r_{1}-L_{E G}-\angle E \times r_{3}-L_{A N i}-L_{E N i}=0\end{array}\right.$

式中 $L_{T}$ 一一轮椅机器人履带总长度

$\angle I$ 一一履带支撑件 $I$ 外侧包围履带段所对圆 心角

$L_{I J}$ 一一履带支撑件 $I 、 J$ 外侧切线段履带长度

$r_{1}$ 一一压带轮半径

$r_{2}$ 一前承重轮、前摆臂行星轮、诱导轮 半径

\section{3 动力学模型}

\section{1 动力学方程}

针对等效串联机器人, 可以利用拉格朗日方法 推导出对于轮椅机器人同样有效的动力学方程。首 先, 系统拉格朗日动力学方程的表达式为 ${ }^{[14]}$

$$
\frac{\mathrm{d}}{\mathrm{d} t} \frac{\partial \boldsymbol{L}}{\partial \dot{\boldsymbol{q}}}-\frac{\partial \boldsymbol{L}}{\partial \boldsymbol{q}}=\boldsymbol{Q}
$$

式中 $\boldsymbol{L}$-一系统拉格朗日函数

$Q$ 一一系统主动广义力

方程中拉格朗日函数 $\boldsymbol{L}$ 可以由系统动能 $E_{K}$ 与 势能 $E_{P}$ 表示为

$$
\boldsymbol{L}=E_{K}-E_{P}
$$

对于等效串联机器人, 其动能与势能可分别表 示为

$$
E_{K}(\boldsymbol{q}, \dot{\boldsymbol{q}})=\frac{1}{2} \dot{\boldsymbol{q}}^{\mathrm{T}} \boldsymbol{D}(\boldsymbol{q}) \dot{\boldsymbol{q}}=\frac{1}{2} \dot{\boldsymbol{q}}^{\mathrm{T}} \times
$$

$\left[\sum_{i=1}^{5}\left\{m_{i} \boldsymbol{J}_{v i}(\boldsymbol{q})^{\mathrm{T}} \boldsymbol{J}_{v i}(\boldsymbol{q})+\boldsymbol{J}_{w i}(\boldsymbol{q})^{\mathrm{T}} \boldsymbol{R}_{i}(\boldsymbol{q}) \boldsymbol{I}_{i} R_{i}(\boldsymbol{q})^{\mathrm{T}} \boldsymbol{J}_{w i}(\boldsymbol{q})\right\}\right] \dot{\boldsymbol{q}}$

$$
E_{P}(\boldsymbol{q})=\sum_{i=1}^{5} m_{i} \boldsymbol{g}^{\mathrm{T}} \boldsymbol{r}_{c i}
$$

式中 $m_{i}, \boldsymbol{I}_{i}$ 一各连杆的质量与惯量矩阵

$\boldsymbol{J}_{v i}, \boldsymbol{J}_{w i}$ 一各连杆的平动与转动雅可比矩阵

$\boldsymbol{R}_{i}$ 一坐标系 $\{i\}$ 相对于坐标系 $\{0\}$ 的旋转 矩阵

$g$ 一重力加速度矢量

$\boldsymbol{r}_{c i}$ 一各连杆质心位置矢量

进而, 系统的拉格朗日方程最终形式可以表 示为

$$
D(\boldsymbol{q}) \ddot{\boldsymbol{q}}+C(\boldsymbol{q}, \dot{\boldsymbol{q}}) \dot{\boldsymbol{q}}+G(\boldsymbol{q})=\boldsymbol{Q}
$$

方程最终形式的系数矩阵的求取过程为

$$
\begin{gathered}
C_{k j}=\sum_{i=1}^{5} \frac{1}{2}\left\{\frac{\partial D_{k j}}{\partial q_{i}}+\frac{\partial D_{k i}}{\partial q_{j}}-\frac{\partial D_{i j}}{\partial q_{k}}\right\} \dot{\boldsymbol{q}}_{i} \\
G_{k}=\frac{\partial E_{P}}{\partial q_{k}}
\end{gathered}
$$

对于拉格朗日方程中的主动广义力, 根据等效 串联机器人中所定义的关节变量, 可以由机器人各 关节所受主动广义力表示为

$$
\boldsymbol{Q}=\left(\begin{array}{lllll}
Q_{x_{A}^{0}} & Q_{z_{A}^{0}} & Q_{\theta} & Q_{\alpha} & Q_{\beta}
\end{array}\right)^{\mathrm{T}}
$$

同时, 由于机器人各关节主动广义力是由履带 与楼梯接触点处的切向力与法向力 (图 2), 以及各履 带轮两侧的履带张紧力产生, 因此, 在假定相邻履 带轮间的履带段内张紧力大小不变的基础上 ${ }^{[17]}$, 拉 格朗日方程中的主动广义力可以由轮椅机器人所受 外部作用力表示为

$$
\left(\begin{array}{c}
Q_{x_{A}^{0}} \\
Q_{z_{A}^{0}} \\
Q_{\theta} \\
Q_{\alpha} \\
Q_{\beta}
\end{array}\right)=\left(\begin{array}{c}
\sum_{i=0}^{n+1}\left(F_{N i}^{x_{0}}+N_{N i}^{x_{0}}\right) \\
\sum_{i=0}^{n+1}\left(F_{N i}^{z_{0}}+N_{N i}^{z_{0}}\right) \\
\sum_{i=0}^{n+1}\left(M_{F_{N i}}^{y_{0}}+M_{N_{N i}}^{y_{0}}\right) \\
\sum_{j=1}^{2} M_{T_{D j}}^{y_{2}}+M_{B} \\
\left(M_{F_{N i}}^{y_{3}}+M_{N_{N i}}^{y_{3}}\right)+\sum_{j=1}^{2} M_{T_{j}}^{y_{3}}+M_{C}
\end{array}\right)
$$

式中 $F_{N i}^{x(z)}, N_{N i}^{x(z)}$ ——轮椅机器人在楼梯接触点处 所受切向力与法向力的 $X(Z)$ 轴分量

$M_{F_{N i}}^{y}, M_{N_{N i}}^{y}$ 一一轮椅机器人在楼梯接触点处 所受切向力与法向力对 $Y$ 轴 的力矩

$M_{T_{D j}}^{y}, M_{T_{E j}}^{y}$ 一一轮椅机器人前、后摆臂行星 轮两侧履带张紧力对 $Y$ 轴的 力矩

$M_{B}, M_{C}$ 一一轮椅机器人前、后摆臂的驱 动力矩

$n$-一楼梯台阶级数

\section{2 主动构件驱动力}

轮椅机器人的主动构件有驱动轮(后摆臂行星 轮)和前、后摆臂。主动构件所需驱动力矩的大小对 机器人机构设计工作有重要的指导意义。

根据轮椅机器人整体受力情况, 驱动轮的驱动 力矩可以表示为

$$
M_{E}=\sum_{i=0}^{n}\left|\boldsymbol{F}_{N i}\right| \cdot r_{3}
$$


同时, 由系统主动广义力表达式(7)可以得到前 摆臂的驱动力矩表达式为

$$
M_{B}=Q_{\alpha}-\sum_{j=1}^{2} M_{T_{D j}}^{y_{4}}
$$

后摆臂的驱动力矩表达式为

$$
M_{C}=Q_{\beta}-\left(M_{F_{N i}}^{y_{5}}+M_{N_{N i}}^{y_{5}}\right)-\sum_{j=1}^{2} M_{T_{E j}}^{y_{5}}
$$

在利用 $(8) \sim(10)$ 对主动构件驱动力矩进行求解 的过程中, 主动广义力 $\boldsymbol{Q}$ 可以在设定机器人部分机 构参数与运动状态的情况下由拉格朗日方程式(6) 求解, 前摆臂行星轮两侧的履带张紧力作为主动控 制量现阶段可以人为给定, 但轮椅机器人在楼梯各 接触点处受到的外部作用力不能全部确定, 因此, 驱动轮与后摆臂的驱动力矩无法精确求取。

由于本文分析的主要目标是为轮椅机器人机 构设计提供理论依据。在这一前提下, 机器人运动 过程中主动构件可能需要的最大驱动力矩的参考意 义最大。因此, 本文对主动构件驱动力矩采用了优 化求取方法。

在所建立的优化模型中, 优化变量如下: 机器人 主动构件驱动力矩 $M_{A}, M_{B}, M_{C}$; 地面作用力 $F_{N i}$, $N_{N i}$; 后摆臂行星轮左、右两侧履带张紧力 $T_{E 1}, T_{E 2}$ 以及前摆臂行星轮上、下两侧履带张紧力 $T_{D 1}, T_{D 2}$ 。

目标函数

$$
\max f(x)=M_{A}, M_{B}, M_{C}
$$

约束方程: 外部作用力约束方程式(7), 驱动轮 驱动力矩约束方程式(8), 前、后摆臂驱动力矩约束 方程式(9)、(10), 同时可建立前、后摆臂履带张紧 力约束方程 ${ }^{[15]}$

$$
T_{E 1}=T_{E 2}+\frac{M_{E}}{r_{3}}-F_{N i}=T_{D 1}=T_{D 2}
$$

地面作用力物理约束方程

$$
N_{N i}^{z^{0}} \geqslant 0 \quad \frac{F_{N_{0}}}{N_{N_{0}}} \leqslant f_{N} \quad \frac{F_{N n}}{N_{N n}} \leqslant f_{N}
$$

式中, $f_{N}$ 为楼梯底面和顶面与轮椅机器人履带间的 最大附着系数。

在优化求解过程中, 针对爬越楼梯过程的不同 阶段, 首先给定机器人的初始位置与履带形状以及 阶段内的运动状态, 并将其代入几何约束方程式 (1) (5)求解出爬越过程各时刻的关节变量 $\boldsymbol{q}$, 进而 利用数值微分方法计算出 $\dot{\boldsymbol{q}}, \ddot{\boldsymbol{q}}$ 。之后将各时刻的 $\boldsymbol{q}, \dot{\boldsymbol{q}}, \ddot{\boldsymbol{q}}$ 代入拉格朗日方程式(6)求解出系统主动
广义力 $\boldsymbol{Q}$ 。最后在给定前摆臂两侧履带张紧力的情 况下, 利用所建立的优化模型优化出各主动构件所 需驱动力矩最大值的变化情况, 并将其作为瞬时驱 动力矩的变化情况。

\section{4 越障过程仿真}

首先设定楼梯参数为 $h=17 \mathrm{~mm}, \varphi=26^{\circ}$, $f_{N}=0.3$ 。对于轮椅机器人, 对应于所定义的等效 串联机器人设定连杆 1 质心在坐标面 $O_{1} X_{1} Z_{1}$ 的位置 为 $(-0.4 \mathrm{~m}, 0.3 \mathrm{~m})$, 其他连杆质心在各自形心处, 其 他参数见下表。

表 轮椅机器人机构参数

\begin{tabular}{ll||cc}
\hline \multicolumn{1}{c||}{ 参数 } & 数值 & 参数 & 数值 \\
\hline$A B$ 间距 $l_{A B} / \mathrm{m}$ & 0.94 & 连杆 1 质量 $m_{1} / \mathrm{kg}$ & 110.0 \\
$A C$ 间距 $l_{A C} / \mathrm{m}$ & 0.69 & 连杆 2 质量 $m_{2} / \mathrm{kg}$ & 2.0 \\
$B E$ 间距 $l_{B E} / \mathrm{m}$ & 0.37 & 连杆 3 质量 $m_{3} / \mathrm{kg}$ & 2.5 \\
$C D$ 间距 $l_{C D} / \mathrm{m}$ & 0.53 & 连杆 4 质量 $m_{4} / \mathrm{kg}$ & 1.0 \\
$F G$ 间距 $l_{F G} / \mathrm{m}$ & 0.65 & 连杆 5 质量 $m_{5} / \mathrm{kg}$ & 15.0 \\
压带轮半径 $r_{1} / \mathrm{m}$ & 0.20 & 连杆 1 惯量 $I_{1} /\left(\mathrm{kg} \cdot \mathrm{m}^{2}\right)$ & 12.000 \\
诱导轮半径 $r_{2} / \mathrm{m}$ & 0.07 & 连杆 2 惯量 $I_{2} /\left(\mathrm{kg} \cdot \mathrm{m}^{2}\right)$ & 0.050 \\
驱动轮半径 $r_{3} / \mathrm{m}$ & 0.03 & 连杆 3 惯量 $I_{3} /\left(\mathrm{kg} \cdot \mathrm{m}^{2}\right)$ & 0.030 \\
履带长度 $L / \mathrm{m}$ & 3.70 & 连杆 4 惯量 $I_{4} /\left(\mathrm{kg} \cdot \mathrm{m}^{2}\right)$ & 0.003 \\
& & 连杆 5 惯量 $I_{5} /\left(\mathrm{kg} \cdot \mathrm{m}^{2}\right)$ & 0.300 \\
\hline
\end{tabular}

仿真过程中, 对于爬越楼梯过程第二阶段前 期, 给定轮椅机器人后摆臂转角为 $\beta=100^{\circ}$, 前承 重轮初始位置为 $x_{A}^{0}=1.45 \mathrm{~m}$, 且在运动过程中保持 速度 $v_{A}^{0}=-\dot{x}_{A}^{0}$ 恒定。通过仿真得到各主动构件驱动 力矩变化见图 $4 \mathrm{a} \sim 4 \mathrm{c}$ 。

对于爬越楼梯过程第二阶段后期, 给定轮椅机 器人座椅倾角为 $\theta=-5^{\circ}$, 前承重轮初始位置为 $x_{A}^{0}=0.7 \mathrm{~m}$, 且在运动过程中保持速度 $v_{A}^{0}=-\dot{x}_{A}^{0}$ 恒 定。通过仿真得到各主动构件驱动力矩变化如图 $4 \mathrm{~d} \sim 4 \mathrm{f}$ 。

对于爬越楼梯过程第三阶段, 给定轮椅机器人 后摆臂转角为 $\beta=140^{\circ}$, 前承重轮初始位置为楼梯 第一级台阶支点处, 且在运动过程保持加速度 $a_{A}=-\ddot{x}_{A}^{0}$ 恒定。通过仿真得到各主动构件驱动力矩 变化如图 $4 \mathrm{~g} \sim 4 \mathrm{i}$ 所示。

对于爬越楼梯过程第四阶段后期, 给定轮椅机 器人座椅倾角为 $\theta=-5^{\circ}$, 前承重轮初始位置为楼梯 第二级与第三级台阶支点之间, 且在运动过程中保 持速度 $v_{A}^{0}=-\dot{x}_{A}^{0}$ 恒定。通过仿真得到各主动构件驱 动力矩变化如图 $4 \mathrm{j} \sim 41$ 所示。

通过仿真结果可以得到在不同运动状态与履带 
张紧力的影响下, 轮椅机器人各主动构件驱动力矩在 爬越楼梯各阶段的变化情况。驱动力矩在各阶段
的变化情况及变化过程中的最大值能够为轮椅机器 人的驱动系统与机械结构设计等工作提供理论基础。

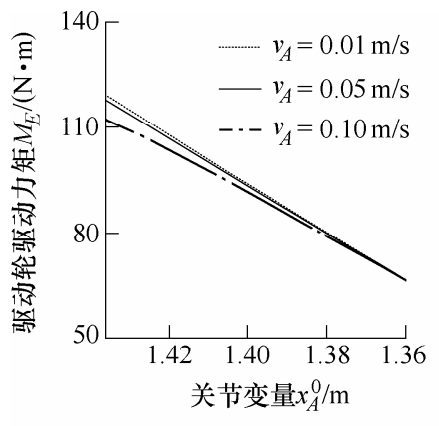

(a) 第二阶段前期驱动轮力矩

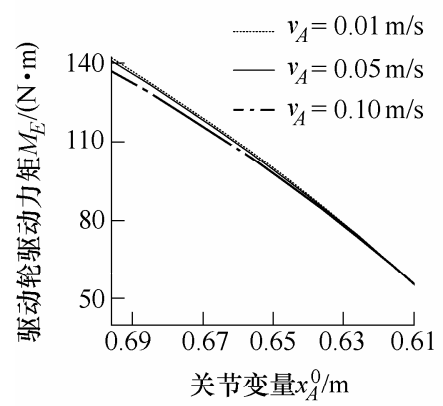

(d) 第二阶段后期驱动轮力矩

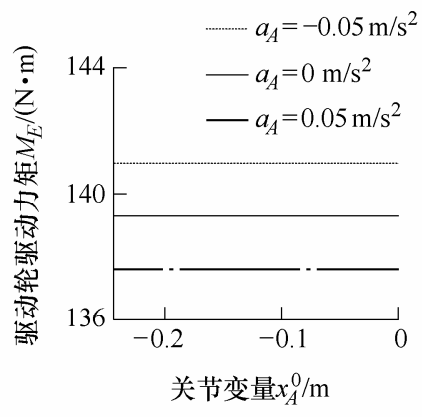

(g) 第三阶段驱动轮力矩

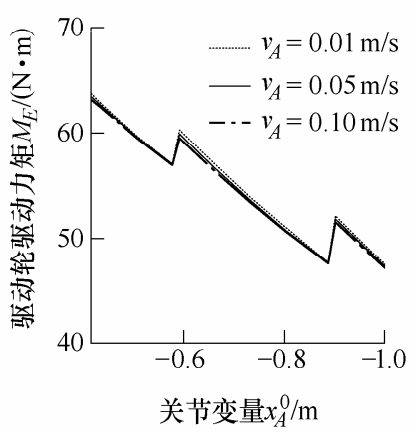

(i) 第四阶段驱动轮力矩

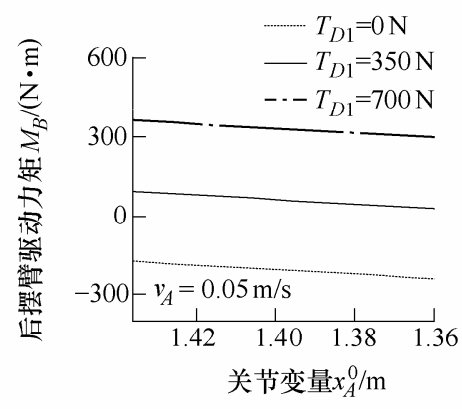

(b) 第二阶段前期后摆臂力矩

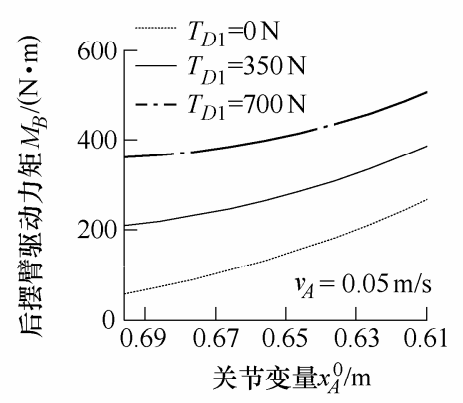

(e) 第二阶段后期后摆臂力矩

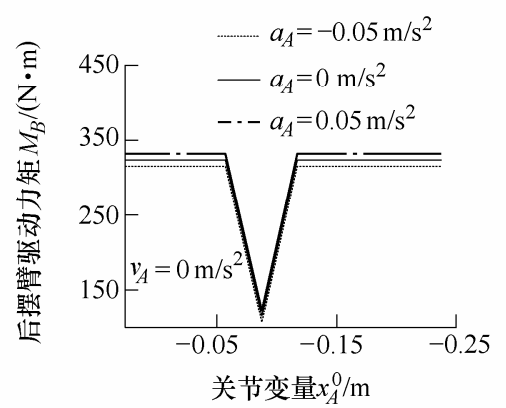

(h) 第三阶段后摆臂力矩

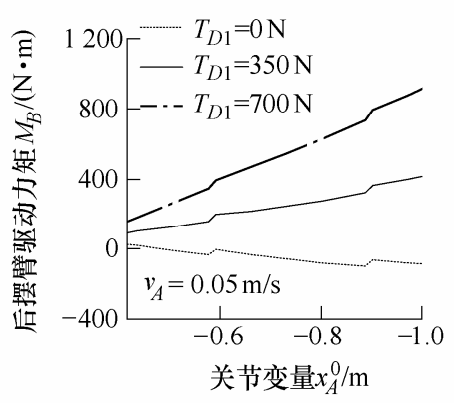

(k) 第四阶段后摆臂力矩

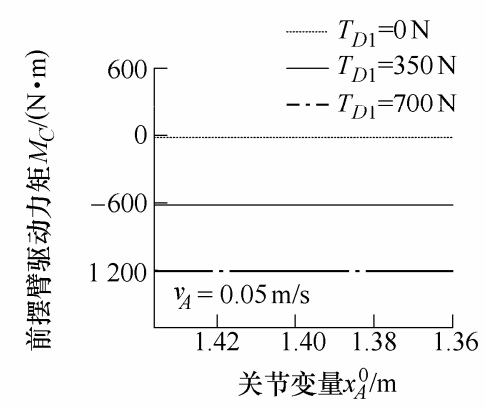

(c) 第二阶段前期前摆臂力矩

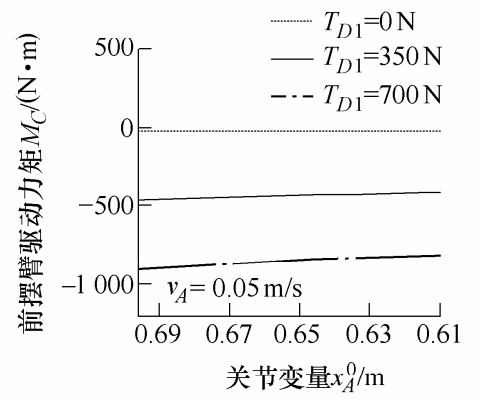

(f) 第二阶段后期前摆臂力矩

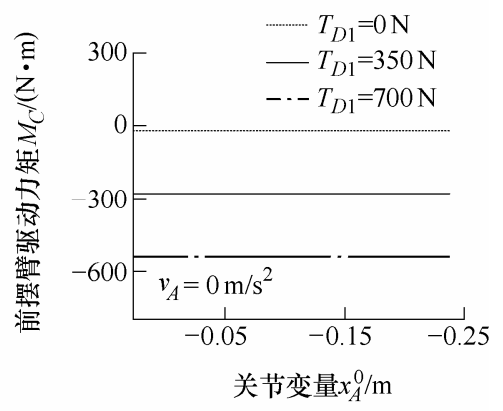

(i) 第三阶段前摆臂力矩

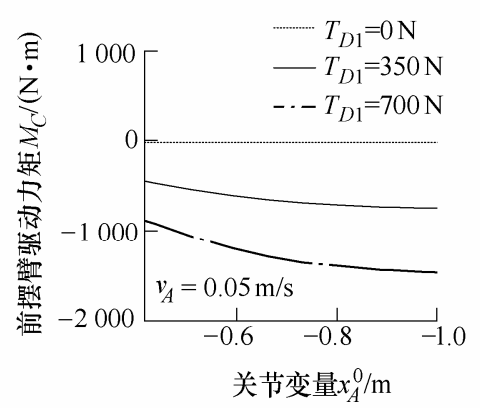

(1) 第四阶段前摆臂力矩

图 4 机器人爬越楼梯过程仿真结果

在轮椅机器人样机爬越楼梯的试验中，楼梯参

\section{5 样机试验}

根据仿真结果进行驱动系统与机械结构设计 之后制作出的轮椅机器人原理样机如图 5 所示。
数与仿真中所设定参数一致, 机器人的行走及前、 后摆臂的转动均由操作者通过操作杆进行手动控 制。试验过程如图 6 所示, 由倾角传感器检测到的 机器人座椅倾角变化如图 7 所示。 


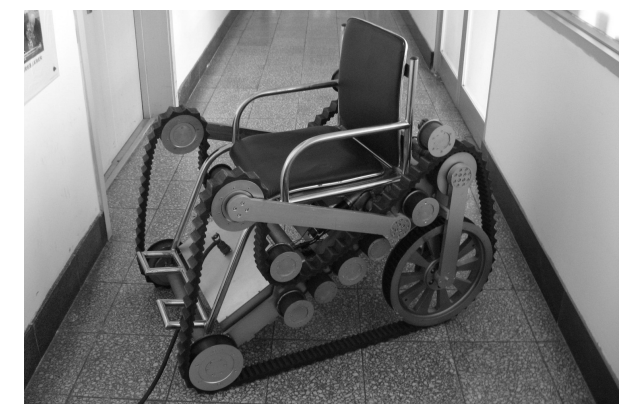

图 5 轮椅机器人原理样机

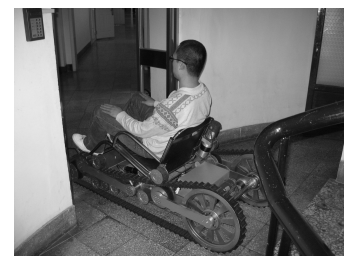

(a)

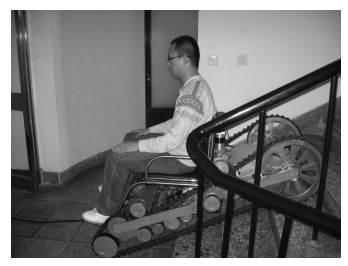

(c)

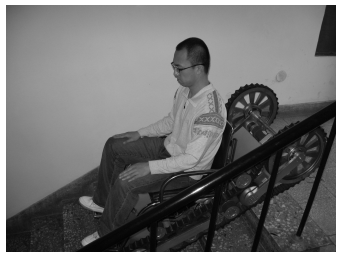

(e)

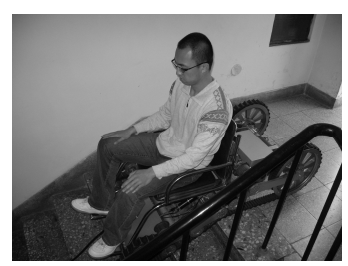

(g)

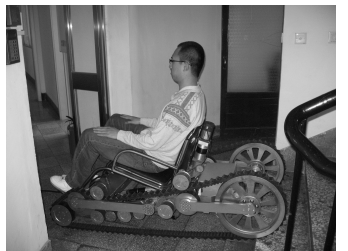

(b)

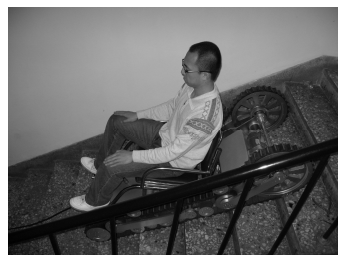

(d)

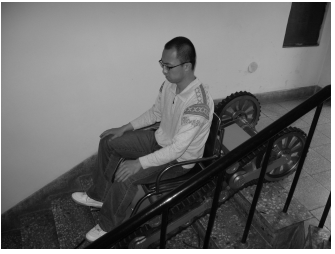

(f)

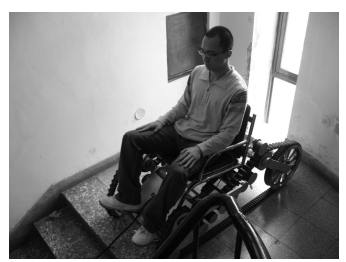

(h)
图 6 轮椅机器人爬越楼梯过程截图

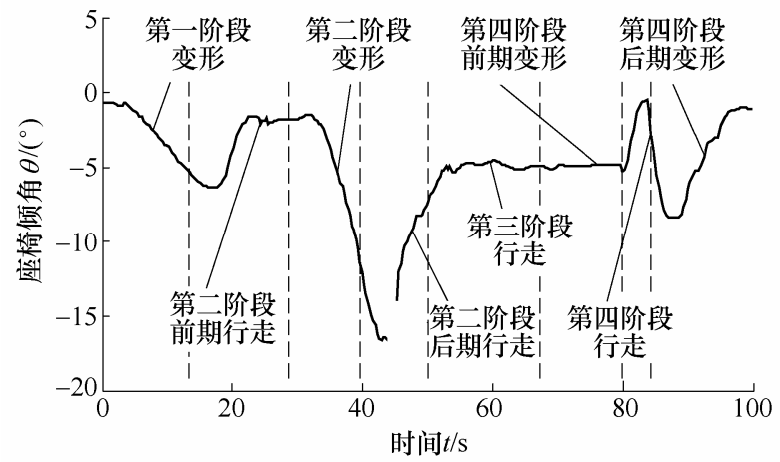

图 7 轮椅机器人爬越楼梯过程座椅倾角变化

从图 6 可以看到, 这种新型轮椅机器人顺利完
成了爬越楼梯作业。从图 7 可以看到, 手动控制情 况下机器人在第四阶段行走过程中利用 $5 \mathrm{~s}$ 的时间 使座椅倾角平稳完成了 $7^{\circ}$ 的变化。这些试验结果 初步验证了机器人的越障能力。同时, 试验结果也 反映出机器人存在爬越楼梯所需时间较长, 爬越过 程座椅倾角的小范围波动与停顿较大等问题。这些 问题主要由现阶段机器人行走及履带形状与张紧力 的手动控制所引起, 将在后续的机器人履带张紧力 的优化及自主爬越楼梯控制等工作中加以解决。

\section{6 结论}

（1）提出了一种采用单节变形履带机构的新型 轮椅机器人机构方案。这种机器人能够对履带形状 与张紧力进行主动控制, 提高了履带式轮椅机器人 对楼梯顶端等障碍的适应能力。

(2) 针对爬越楼梯这种越障运动, 将轮椅机器 人转化为等效串联机器人。在所建立的几何模型与 动力学模型的基础上, 采用优化的方法实现了对机 器人主动构件驱动力矩的求解。

(3) 利用仿真得到的轮椅机器人爬越楼梯过程 主动构件驱动力矩变化情况, 指导完成了机器人最 终设计与样机制作。样机爬越楼梯试验的顺利完成 验证了机器人的越障能力。

(4) 后续研究工作将集中在轮椅机器人履带张 紧力优化与自主爬越楼梯控制等方面。

\section{参 考 文 献}

[1] UUSTAL H, MINKEL J L. Study of the independence IBOT 3000 mobility system: An innovative power mobility device, during use in community environments[J]. Archives of Physical Medicine and Rehabilitation, 2004, 85(12): 2002-2010.

[2] COX K. Battery powered stair-climbing wheelchair: US, 6484829 B1[P]. 2002-11-26.

[3] LAWN $\mathrm{M} \mathrm{J}$, ISHIMATSU $\mathrm{T}$. Modeling of a stair-climbing wheelchair mechanism with high single-step capability[J]. IEEE Transactions on Neural System and Rehabilitation Engineering, 2003, 11(3): 323-332.

[4] MORALES R, GONZALES A, FELIU $\mathrm{V}$, et al. Environment adaptation of a new staircase-climbing wheelchair[J]. Autonomous Robots, 2007, 23: 275-292.

[5] YUAN Jianjun, HIROSE S. Research on leg-wheel hybrid stair-climbing robot, zero carrier[C]// Proceedings of the IEEE International Conference on Robotics and 
Biomimetics, August 22-26, 2004, Shenyang, China. Shenyang: IEEE, 2004: 654-659.

[6] Garaventa Lift Co. Ltd. Emergency evacuation chair[EB/OL]. [2012-11-12]. http: //www.garaventalift. vcom/en/products/evacuation_chairs.html.

[7] LAWN M J, SAKAI T, KUROIWA $\mathrm{M}$, et al. Development and practical application of a stairclimbing wheelchair in Nagasaki[J]. Journal of HWRS-ERC, 2001, 2(2): 33-39.

[8] TGR Co. Ltd. Explorer[EB/OL]. [2012-11-12]. http: //www. tgr.it/ prodotti-explorer-en.html.

[9] LSFFONT I, GUILLON B, FERMANIAN C, et al. Evaluation of a stair-climbing power wheelchair in 25 people with tetraplegia[J]. Archives of Physical Medicine and Rehabilitation, 2008, 89(10): 1958-1964.

[10] MURPHY R R. Marsupial and shape-shifting robots for urban search and rescue[J]. IEEE Intelligent Systems and Their Applications, 2000, 15(2): 14-19.

[11] LIU Yugang, LIU Guangjun. Track-stair Interaction analysis and online tipover prediction for a self-reconfigurable tracked mobile robot climbing stairs[J]. IEEE/ASME Transactions on Mechatronics, 2009, 14(5): 528.
[12] LIM S K, PARK D I, KWAK Y K, et al. Variable geometry single-tracked mechanism for a rescue robot $[\mathrm{C}] / /$ Proceedings of the 2005 IEEE International Workshop on Safety, Security and Rescue Robotics, June 2005, Kobe, Japan, 2005: 111-115.

[13] PAILLAT J, LUCIDARME P, HARDOUIN L. Original design of unmamed ground vehicle (UGV) for exploration in rough terrain[J]. Advanced Robotics, 2010, 24: 255-276.

[14] 谭民, 徐德, 侯增广, 等. 先进机器人控制 [M]. 北京: 高等教育出版社, 2007 .

TAN Min, XU De, HOU Zengguang, et al. Advanced robot control[M]. Beijing: Higher Education Press, 2007.

[15] HUN K, HONG D. Track tension estimation in track vehicle under various maneuvering tasks[J]. Journal of Dynamic Systems, Measurement, and Control, 2001, 123(6): 179-185.

作者简介: 于苏洋(通信作者), 男, 1984 年出生, 博士研究生。主要研 究方向为机器人机构设计与分析。

E-mail: yusuyang@sia.cn

王挺, 男, 1978 年出生, 博士, 副研究员。主要研究方向为机器人控制。 E-mail: wangting@sia.cn 